\title{
Well-Posedness of Difference Elliptic Equation
}

\author{
PAVEL E. SOBOLEVSKII \\ Institute of Mathematics, Hebrew University, Givat Ram, 91904 Jerusalem, Israel
}

(Received 15 November 1996; Revised 23 January 1997)

\begin{abstract}
The exact with respect to step $h \in(0,1]$ coercive inequality for solutions in $C^{h}$ of difference elliptic equation is established.
\end{abstract}

Keywords: Coercive inequalities, Properties of positivity

\section{INTRODUCTION}

It is well-known in the theory of differential equations that the coercive inequalities approach appeared to be very useful for the investigation of general boundary value problems for elliptic and parabolic differential equations.

The coercive inequalities hold also for various difference analogues of such problems. These inequalities, evidently, permit to prove not only the existence of solutions but also well-posedness of these problems. Main role of the coercive inequalities for difference problems lies in that they present a special type of stability, which permits the existence of exact, i.e. two-sided estimates of the rate of convergence approximate solutions (with respect to the corresponding coercive norms).

As it turns out, there are situations when the difference problems are well-posed, but their limit variants - differential problems - are ill-posed. This paper deals with a consideration just of one of such cases. The established here exact (with respect to step $h$ of difference scheme) coercive inequality gives the possibility to find (almost) exact estimates of the rate of convergence of approximate solutions in the case when the differential problem is ill-posed.

\subsection{Ill-Posedness of Differential Elliptic Equation in $C$}

We will consider the simplest elliptic differential equation

$$
v-\left(\partial^{2} v / \partial x_{1}^{2}+\partial^{2} v / \partial x_{2}^{2}\right)=f
$$

on the plane $R^{2}$ of points $x=\left(x_{1}, x_{2}\right)$. It is natural to call function $v(x)=v\left(x_{1}, x_{2}\right)$ the (classical) solution of Eq. (0.1), if it has the continuous and bounded partial derivatives till the second order, and if it satisfies Eq. (0.1). We will consider differential equation $(0.1)$ as the operator equation in the Banach space $C=C\left(R^{2}\right)$ of continuous and bounded (scalar) functions $\psi(x)=\psi\left(x_{1}, x_{2}\right)$ with norm

$$
\|\psi\|_{C}=\sup _{x \in R^{2}}|\psi(x)|
$$


For the existence of such solution $v$ of Eq. (0.1), evidently, it is necessary that

$$
f \in C \text {. }
$$

We will say that Eq. (0.1) is well-posed in $C$ (see [1]), if the following two conditions are fulfilled:

$\left(a_{1}\right)$ There exists the unique solution $v(x)=v(x ; f)$ in $C$ of Eq. $(0.1)$ for any $f \in C$. It means, in particular, that formula

$$
[v(f)](x)=v(x ; f)
$$

defines the homogeneous and additive operator, acting from $C$ in the Banach space $C^{2}$ of (scalar) functions $\psi(x)=\psi\left(x_{1}, x_{2}\right)$, having continuous and bounded partial derivatives till the second order, with norm

$$
\begin{aligned}
\|\psi\|_{C^{2}}= & \|\psi\|_{C}+\sum_{i=1}^{2}\left\|\partial \psi / \partial x_{i}\right\|_{C} \\
& +\sum_{i, j=1}^{n}\left\|\partial^{2} \psi / \partial x_{i} \partial x_{j}\right\|_{C}
\end{aligned}
$$

$\left(\mathrm{a}_{2}\right)$ Operator $v(f)$ is continuous in $C$.

This property is, evidently, equivalent to inequality

$$
\|v(f)\|_{C} \leq M \cdot\|f\|_{C}
$$

with some $1 \leq M<+\infty$, does not depend on $f \in C$. It turns out that property $\left(\mathrm{a}_{1}\right)$ leads us to the essentially more stronger inequality. In fact, the acting in $C$ with domain $C^{2}$ operators

$$
\left(A_{i} \psi\right)(x)=-\partial^{2} \psi / \partial x_{1}^{2} \quad(i=1,2),
$$

evidently, are closed. Then from properties $\left(a_{1}\right)$ and $\left(a_{2}\right)$ it follows that superposition operators

$$
\left[A_{i} v(f)\right](x)=-\partial^{2} v(x ; f) / \partial x_{1}^{2} \quad(i=1,2)
$$

are closed operators, defined on the whole Banach space $C$. Therefore, by the Banach theorem, operators $(0.8)$ are bounded. This statement leads us to coercive inequality

$$
\|v\|_{C^{2}} \leq M \cdot\|f\|_{C}
$$

for solutions in $C$ of Eq. (0.1) with some $1 \leq M<+\infty$, does not depend on $f \in C$. However, it is well-known (see [1]) that Eq. (0.1) is not well-posed in $C$. The corresponding counterexample can be given by $(0<\alpha<1)$

$$
\begin{aligned}
& v_{\alpha}(x)=\left(x_{1}^{2}-x_{2}^{2}\right) \cdot \ln ^{\alpha} \frac{1}{x_{1}^{2}+x_{2}^{2}} \\
& \quad\left(0<x_{1}^{2}+x_{2}^{2}<\frac{1}{9}\right), \\
& v_{\alpha}(x)=0 \quad\left(\frac{4}{9} \leq x_{1}^{2}+x_{2}^{2} \text { and } x_{1}=x_{2}=0\right), \\
& v_{\alpha} \in C^{2} \quad\left(\frac{4}{10} \leq x_{1}^{2}+x_{2}^{2}\right) .
\end{aligned}
$$

It means that $v_{\alpha} \in C^{1}$ and for $0 \leq x_{1}^{2}+x_{2}^{2} \leq 1 / 9$

$$
\begin{aligned}
& \partial^{2} v_{\alpha} / \partial x_{1}^{2}=2 \cdot \ln ^{\alpha} \frac{1}{x_{1}^{2}+x_{2}^{2}}+a_{\alpha, 1}(x) \\
& \partial^{2} v_{\alpha} / \partial x_{2}^{2}=-2 \cdot \ln ^{\alpha} \frac{1}{x_{1}^{2}+x_{2}^{2}}+a_{\alpha, 2}(x)
\end{aligned}
$$

for some continuous functions $a_{\alpha, i}(x)=a_{\alpha, i}\left(x_{1}, x_{2}\right)$ $(i=1,2)$. Therefore, evidently, Eq. $(0.1)$ is illposed in $C$. It means that coercive inequality (0.9) is not true for any solution in $C$ of Eq. (0.1).

\subsection{Well-Posedness of Difference Equation in $C^{h}$}

We will consider now the difference analog of differential equation (0.1), namely difference equation

$$
\begin{gathered}
v_{i, j}-\left[\left(v_{i+1, j}-2 \cdot v_{i, j}+v_{i-1, j}\right) \cdot h^{-2}\right. \\
\left.+\left(v_{i, j+1}-2 \cdot v_{i, j}+v_{i, j-1}\right) \cdot h^{-2}\right] \\
=f_{i, j} \quad(i, j=-\overline{\infty,+\infty})
\end{gathered}
$$

for some $0<h \leq 1$. We will consider Eq. (0.12) as operator equation

$$
v^{h}-\left(D^{h, 2} v^{h}+D_{2}^{h, 2} v^{h}\right)=f^{h}
$$


in the Banach space $C^{h}$ of (scalar) bounded grid functions

$$
\psi^{h}=\left(\psi_{i, j} ; i, j=-\overline{\infty,+\infty}\right)
$$

with norm

$$
\left\|\psi^{h}\right\|_{C}=\sup _{i, j=-\infty,+\infty}\left|\psi_{i, j}\right|
$$

Here operators $D_{k}^{h, 2}(k=1,2)$ are defined by formulas

$$
\begin{aligned}
D_{1}^{h, 2} \psi^{h} & =\left[\left(\psi_{i+1, j}-2 \cdot \psi_{i, j}+\psi_{i-1, j}\right) \cdot h^{-2}\right. \\
i, j & =-\overline{\infty,+\infty}], \\
D_{2}^{h, 2} \psi^{h} & =\left[\left(\psi_{i, j+1}-2 \psi_{i, j}+\psi_{i, j-1}\right) \cdot h^{-2}\right. \\
i, j & =-\overline{\infty,+\infty}] .
\end{aligned}
$$

For any $f^{h} \in C^{h}$ Eq. (0.13) has the unique solution $v^{h} \in C^{h}$, and the difference coercive inequality

$$
\left\|v^{h}\right\|_{C^{h}}+\left\|D_{1}^{h, 2}\right\|_{C^{h}}+\left\|D_{2}^{h, 2} v^{h}\right\|_{C^{h}} \leq M_{C}(h) \cdot\left\|f^{h}\right\|_{C^{h}}
$$

takes place with some $1 \leq M_{C}(h)<+\infty$, does not depend on $f^{h} \in C^{h}$. In fact, let us consider more general, then $(0.13)$ operator equation with parameter $\lambda>0$

$$
\lambda v^{h}-\left(D_{1}^{h, 2} v^{h}+D_{2}^{h, 2} v^{h}\right)=f^{h},
$$

or (infinite) system of linear algebraic equations

$$
\begin{aligned}
& \lambda v_{i, j}-\left[\left(v_{i+1, j}-2 \cdot v_{i, j}+v_{i-1, j}\right) \cdot h^{-2}\right. \\
& \left.\quad+\left(v_{i, j+1}-2 \cdot v_{i, j}+v_{i, j-1}\right) \cdot h^{-2}\right]=f_{i, j} \\
& (i, j=\overline{-\infty,+\infty}) .
\end{aligned}
$$

Since, evidently, operators (0.16) are bounded (for fixed $h$ ), then, in virtue of contraction mapping principle, Eq. (0.18) for any $f^{h} \in C^{h}$ has the unique solution $v^{h} \in C^{h}$, if $\lambda>0$ is sufficiently large. Further we apply the maximum principle (to system (0.19)) and obtain a priori estimate

$$
\left\|v^{h}\right\|_{C^{h}} \leq \lambda^{-1}\left\|f^{h}\right\|_{C^{h}}
$$

Therefore Eq. (0.18) has the unique solution $v^{h} \in C^{h}$ for any $f^{h} \in C^{h}$ and $\lambda>0$, i.e. operator $\lambda I-\left(D_{1}^{h, 2}+D_{2}^{h, 2}\right)$ has (bounded) inverse for any $\lambda>0$, and estimate

$$
\left\|\left[\lambda I-\left(D_{1}^{h, 2}+D_{2}^{h, 2}\right)\right]^{-1}\right\|_{C^{h} \rightarrow C^{h}} \leq \lambda^{-1}
$$

is true. Since $D_{k}^{h, 2}(k=1,2)$ are bounded operators (for fixed $h$ ), then coercive inequality $(0.17)$ is true. The value $M_{C}(h)$ in this inequality, evidently, must tend to $+\infty$, when $h \rightarrow+0$, since the differential coercive inequality $(0.9)$ is not true. It is the consequence of ill-posedness in $C$ of differential equation (0.1). From estimate $(0.21)$ and from formulas (0.16), evidently, it follows that we can put

$$
M_{C}(h)=M \cdot h^{-2}
$$

in inequality (0.17) for some $1 \leq M<+\infty$, does not depend on $f^{h} \in C^{h}$ and $0<h \leq 1$. It turns out that essentially more exact result is true. Namely, for solution $v^{h}$ in $C^{h}$ of Eq. (0.13) coercive inequality $(0.17)$ takes place for

$$
M_{C}(h)=M_{0} \cdot \ln 1 / h \quad\left(0<h \leq \frac{1}{2}\right)
$$

with some $1 \leq M_{0}<+\infty$, does not depend on $f^{h}$ and $h$. It is, in particular, the consequence of theory of difference equations which is devoted in this paper. Formula (0.23) means that

$$
\begin{aligned}
& \sup _{f^{h} \in C^{h} \cdot f^{h} \neq 0}\left[\left\|D_{1}^{h, 2} v^{h}\right\|_{C^{h}}+\left\|D_{2}^{h, 2} v^{h}\right\|_{C^{h}}\right] \cdot\left\|f^{h}\right\|_{C^{h}}^{-1} \\
& \quad \leq M_{0} \cdot \ln 1 / h .
\end{aligned}
$$

It turns out that $(0.24)$ is the exact with respect to order $h \rightarrow+0$ estimate. In fact (see formula $(0.10)$ ), let

$v_{i, j}=v_{1}\left(x_{1}, x_{2}\right) \quad\left(x_{1}=i h, x_{2}=j h ; i, j=\overline{-\infty,+\infty}\right)$.

Then from formulas $(0.11)$ it follows that $(0<$ $\left.x_{1}^{2}+x_{2}^{2}<\frac{1}{9}\right)$ 


$$
\begin{aligned}
& \left(v_{i+1, j}-2 \cdot v_{i, j}+v_{i-1, j}\right) \cdot h^{-2} \\
& =\int_{0}^{1} y\left[\int _ { - 1 } ^ { 1 } \left[2 \cdot \ln \frac{1}{\left(x_{1}+h y z\right)^{2}+x_{2}^{2}}\right.\right. \\
& \left.\left.\quad+a_{1,1}\left(x_{1}+h y z, x_{2}\right)\right] \mathrm{d} z\right] \mathrm{d} y, \\
& \left(v_{i, j+1}-2 \cdot v_{i, j}+v_{i, j-1}\right) \cdot h^{-2} \\
& =\int_{0}^{1} y\left[\int _ { - 1 } ^ { 1 } \left[-2 \cdot \ln \frac{1}{x_{1}^{2}+\left(x_{2}+h y z\right)^{2}}\right.\right. \\
& \left.\left.\quad+a_{1,2}\left(x_{1}, x_{2}+h y z\right)\right] \mathrm{d} z\right] \mathrm{d} y
\end{aligned}
$$

Therefore for some $a_{0}>0$ and sufficiently small $h>0$ estimates from below

$$
\begin{aligned}
& \left|\left(v_{i+1, j}-2 \cdot v_{i, j}+v_{i-1, j}\right) \cdot h^{-2}\right| \\
& \left|\left(v_{i, j+1}-2 \cdot v_{i, j}+v_{i, j-1}\right) \cdot h^{-2}\right| \\
& \quad \geq 8 \cdot\left(1-a_{0} h\right) \cdot \ln 1 / h
\end{aligned}
$$

are true. Finally, from $(0.10)$ and $(0.26)$ it follows that estimates from above

$$
\begin{aligned}
\left|f_{i, j}\right| & \leq m_{0}, f_{i, j}=v_{i, j}-\left[\left(v_{i+1, j}-2 \cdot v_{i, j}+v_{i-1, j}\right) \cdot h^{-2}\right. \\
& \left.+\left(v_{i, j+1}-2 \cdot v_{i, j}+v_{i, j-1}\right) \cdot h^{-2}\right]
\end{aligned}
$$

take place for some $0<m_{0}<+\infty$, does not depend on $h$. Therefore from $(0.27)$ and $(0.28)$ it follows that estimate from below

$$
\begin{aligned}
& \sup _{f^{h} \in C^{h}, f^{h} \neq 0}\left[\left\|v^{h}\right\|_{C^{h}}+\mid D_{1}^{h, 2} v^{h} \|_{C^{h}}\right. \\
& \left.\quad+\left\|D_{2}^{h, 2} v^{h}\right\|_{C^{h}}\right] \cdot\left\|f^{h}\right\|_{C^{h}}^{-1} \geq \frac{8-a_{0} h}{m_{0}} \cdot \ln 1 / h
\end{aligned}
$$

holds for sufficiently small $h>0$.

\subsection{The Almost Exact Estimate of Convergence Rate}

Let $v$ be the solution in $C$ of Eq. (0.1), having the continuous and bounded partial derivatives till the fourth order. Let further $v_{i, j}(i, j=\overline{-\infty,+\infty})$ be the solution of system $(0.12)$ for

$$
f_{i, j}=f(i h, j h)
$$

Then, evidently, values

$$
z_{i, j}=v(i h, j h)-v_{i, j} \quad(i, j=\overline{-\infty,+\infty})
$$

are the solutions of the system

$$
\begin{aligned}
& z_{i, j}-\left[\left(z_{i+1, j}-2 \cdot z_{i, j}+z_{i-1, j}\right) h^{-2}\right. \\
& \left.\quad+\left(z_{i, j+1}-2 \cdot z_{i, j}+z_{i, j-1}\right) \cdot h^{-2}\right]=\Gamma_{i, j},
\end{aligned}
$$

and for values $\Gamma_{i, j}$ estimates

$$
\left|\Gamma_{i, j}\right| \leq M \cdot h^{2}
$$

take place for some $1 \leq M<+\infty$, does not depend on $h$. Therefore, from $(0.24)$ it follows that estimate from above

$$
\begin{aligned}
& \left\|z^{h}\right\|_{C^{h}}+\left\|D_{1}^{h, 2} z^{h}\right\|_{C^{h}}+\left\|D_{2}^{h, 2} z^{h}\right\|_{C^{h}} \\
& \quad \leq M_{1} \cdot h^{2} \cdot \ln 1 / h
\end{aligned}
$$

is true for some $1 \leq M_{1}<+\infty$, does not depend on $h$.

Finally, let $f\left(x_{1}, x_{2}\right) \not \equiv 0$ be the smooth function, which partial derivatives till the second order sufficiently quickly tend to zero, when $x_{1}^{2}+x_{2}^{2}$ tend to infinity. Then, evidently,

$$
\sup _{x \in R^{2}}\left|\partial^{4} v / \partial x_{1}^{4}+\partial^{4} v / \partial x_{2}^{4}\right|>0
$$

and therefore estimate

$$
\sup _{k, j=-\infty,+\infty}\left|\Gamma_{i, j}\right| \geq m \cdot h^{2}
$$

is true for some $0<m<+\infty$, does not depend on $h$. Estimate (0.36) and triangle inequality lead us to estimate from below

$$
\left\|z^{h}\right\|_{C^{h}}+\left\|D_{1}^{h, 2} z^{h}\right\|_{C^{h}}+\left\|Z^{h}+\right\| D_{2}^{h, 2} \|_{C^{h}} \geq m_{1} h^{2}
$$


for some $0<m_{1}<+\infty$, does not depend on $h$. Estimates (0.34) and (0.37) give the almost exact estimate of convergence rate of difference method $(0.12)$ in the difference coercive norm.

\subsection{The Content of Paper}

This paper is devoted to investigation of wellposedness of differential equation

$$
-\mathrm{d}^{2} v / \mathrm{d} t^{2}+A v=f \quad(-\infty<t<+\infty)
$$

and its difference analog

$$
\begin{aligned}
& -\left(v_{i+1}-2 \cdot v_{i}+v_{i-1}\right) \cdot h^{-2}+A v_{i}=f_{i} \\
& \quad(i=\overline{-\infty,+\infty})
\end{aligned}
$$

in the arbitrary Banach space $E$. Here $A$ is the (unbounded) closed linear operator in $E$ with dense domain $D(A)$. Equation (0.38) is considered in the functional (abstract) Hölder space $C^{\alpha}(E)(0<\alpha<1)$, and for any positive (see [2]) in $E$ operator $A$ coercive inequality

$$
\|A v\|_{C^{\alpha}(E)} \leq M \cdot \alpha^{-1} \cdot(1-\alpha)^{-1} \cdot\|f\|_{C^{\alpha}(E)}
$$

is established for its solution $v$ in $C^{\alpha}(E)$ with some $1 \leq M<+\infty$, does not depend on $f \in C^{\alpha}(E)$ and $0<\alpha<1$. To differential equation (0.38) Grisvard's theory (see [3]) is applicable, but it leads us to the coercive inequality $\left(0<\alpha \leq \frac{1}{2}\right)$

$$
\|A v\|_{C^{\alpha}(E)} \leq M \cdot \alpha^{-2} \cdot\|f\|_{C^{\alpha}(E)} .
$$

Difference equation (0.39) is considered as the operator equation in the Hölder space $C^{h, \alpha}(E)$ $(0<\alpha<1)$ of (abstract) grid functions, and for any strongly positive (see [2]) in $E$ operator $A$ coercive inequality

$$
\left\|A v^{h}\right\|_{C^{h, \alpha}(E)} \leq M \cdot \alpha^{-1}(1-\alpha)^{-1} \cdot\left\|f^{h}\right\|_{C^{h, \alpha}(E)}
$$

is established for its solutions $v^{h}$ in $C^{h, \alpha}(E)$ with some $1 \leq M<\infty$, does not depend not only on $f^{h} \in C^{h, \alpha}(E)$ and $0<\alpha<1$, but also on $h$. From inequality $(0.42)$ it follows that

$$
\left\|A v^{h}\right\|_{C^{h}(E)} \leq M \cdot \ln 1 / h \cdot\left\|f^{h}\right\|_{C^{h}(E)} \quad\left(0<h \leq \frac{1}{2}\right) .
$$

Here $C^{h}(E)$ is the Banach space of uniformly bounded grid functions $\psi^{h}=\left(\psi_{i} \in E\right.$; $i=\overline{-\infty,+\infty})$. Inequality (0.43) leads us to formula $(0.23)$ of exact value $M_{c}(h)$ in difference coercive inequality (0.17).

To difference equation (0.39) Grisvard's theory is also applicable even in more general case, when $A$ is only positive operator in $E$, but it leads us to inequality

$\left\|A v^{h}\right\|_{C^{h, \alpha}(A)} \leq M \cdot \alpha^{-2} \cdot\left\|f^{h}\right\|_{C^{h, \alpha}(E)} \quad(0<\alpha \leq 1 / 2)$.

From (0.44) only estimate

$$
\left\|A v^{h}\right\|_{C^{h}(E)} \leq M \cdot \ln ^{2} 1 / h \cdot\left\|f^{h}\right\|_{C^{h}(E)}
$$

follows.

\section{DIFFERENTIAL EQUATION OF THE SECOND ORDER IN THE BANACH SPACE}

\subsection{Well-Posedness in $C(E)$}

We will consider (abstract) differential equation

$$
-v^{\prime \prime}(t)+A v(t)=f(t) \quad(-\infty<t<+\infty)
$$

in the Banach space $E$ as the operator equation in the functional Banach space $C(E)=C[(-\infty$, $+\infty), E]$ with norm

$$
\|\psi\|_{C(E)}=\sup _{-\infty<t<+\infty}\|\psi(t)\|_{E}
$$

We will call the function $v(t) \in C(E)$ the solution in $C(E)$ of Eq. (1.1), if $v^{\prime \prime}(t), A v(t) \in C(E)$, and 
Eq. (1.1) is fulfilled. If such solution exists, then, evidently,

$$
f(t) \in C(E) .
$$

We will say that Eq. (1.1) is well-posed in $C(E)$, if the following two conditions are fulfilled:

$\left(\mathrm{a}_{1}\right)$ For any $f \in C(E)$ there exists the unique solution $v(t)=v(t ; f)$ in $C(E)$ of Eq. (1.1). It, in particular, means that

$$
\mathrm{d}^{2}[v(t ; f)] /(\mathrm{d} t)^{2} \quad \text { and } \quad A v(t ; f)
$$

are acting in $C(E)$ additive and homogeneous operators, defining on whole Banach space $C(E)$.

$\left(\mathrm{a}_{2}\right)$ Operator $v(t ; f)$ is continuous in $C(E)$, i.e. inequality

$$
\|v(t ; f)\|_{C(E)} \leq M_{s} \cdot\|f\|_{C(E)} \quad\left(1 \leq M_{s}<+\infty\right)
$$

holds.

Properties $\left(a_{1}\right)$ and $\left(a_{2}\right)$, in virtue of Banach's theorem, lead us to coercive inequality

$$
\begin{aligned}
& \left\|v^{\prime \prime}\right\|_{C(E)}+\|A v(t)\|_{C(E)} \leq M_{C} \cdot\|f\|_{C(E)} \\
& \quad\left(1 \leq M_{C}<+\infty\right) .
\end{aligned}
$$

Inequality (1.6) permits to investigate the spectral properties of operator coefficient $A$ for wellposed in $C(E)$ of Eq. (1.1). For any $u \in D(A)$ and $\lambda \geq 0$ we will put

$$
\psi=\lambda u+A u .
$$

Then, evidently, function $\mathrm{e}^{\mathrm{i} \sqrt{\lambda} t} u(\mathrm{i}=\sqrt{-1})$ is the solution in $C(E)$ of Eq. (1.1) for function $f(t)=\mathrm{e}^{\mathrm{i} \sqrt{\lambda}} \psi$. Therefore from coercive inequality (1.6) inequality

$$
\lambda\|u\|_{E}+\|A u\|_{E} \leq M_{C}\|\psi\|_{E}
$$

follows. We will suppose that operator $A$ has bounded in $E$ inverse $A^{-1}$. Then, evidently, from inequality (1.8) it follows that operator $\lambda I+A$ has bounded in $E$ inverse for any $\lambda \geq 0$, and estimate

$$
\left\|(\lambda I+A)^{-1}\right\|_{E \rightarrow E} \leq M \cdot(\lambda+1)^{-1}
$$

is true for some $1 \leq M<+\infty$. Such $A$ is called positive in $E$ operator (see [2]). So, if Eq. (1.1) is well-posed in the functional Banach space $C(E)$, then $A$ is positive operator in the Banach space $E$ (under condition that operator $A^{-1}$ is bounded in $E)$. Whether the positivity of operator $A$ in $E$ is sufficient condition of the well-posedness of Eq. (1.1) in $C(E)$ ?.

For arbitrary Banach space $F$ let us consider the acting in $C(F)=C[(-\infty,+\infty), F]$ operator $A$, defining by formula

$$
A \psi(x)=-\psi^{\prime \prime}(x)+\psi(x)(-\infty,+\infty)
$$

on functions $\psi(x) \in C(F)$, such that $\psi^{\prime \prime}(x) \in C(F)$. Evidently, operator $\lambda I+A$ has the bounded inverse for any $\lambda \geq 0$, and formula

$$
\begin{aligned}
& {\left[(\lambda I+A)^{-1} \psi\right](x)} \\
& \quad=\frac{1}{2 \sqrt{\lambda+1}} \int_{-\infty}^{+\infty} \mathrm{e}^{-\sqrt{1+\lambda}|x-y|} \psi(y) \mathrm{d} y
\end{aligned}
$$

holds. From (1.11) estimate (1.9) (for $M=1$ ) follows, i.e. $A$ is positive operator in the Banach space $E=C(F)$. However the counter-example from the Introduction shows that Eq. (1.1) is illposed in $C(E)$.

\subsection{Formula of Solution in $C(E)$}

From estimate (1.9), evidently, it follows that operator $\lambda I+A$ has bounded inverse for all complex numbers $\lambda=\sigma+\mathrm{i} \tau \in \mathcal{G}_{\varepsilon}^{+}=\mathcal{G}_{\varepsilon}^{+}(M)$ $(0<\varepsilon<1)$, such that

$$
|\tau| \leq \frac{1-\varepsilon}{M}(1+\sigma) \quad(\sigma \geq 0)
$$

or

$$
\left(\sigma^{2}+\tau^{2}\right)^{1 / 2} \leq \frac{1-\varepsilon}{M} \quad(\sigma \leq 0)
$$


and estimate

$$
\left\|(\lambda I-A)^{-1}\right\|_{E \rightarrow E} \leq M_{1} \cdot \varepsilon^{-1} \cdot(1+|\lambda|)^{-1}
$$

holds for some $1 \leq M_{1}<+\infty$, does not depend on $0<\varepsilon<1$. It means that spector $\sigma(A)$ of operator $A$ is outside of set $\mathcal{G}_{\varepsilon}^{-}=-\mathcal{G}_{\varepsilon}^{+}$and inside of $\mathcal{G}_{\varepsilon}^{-}$and on its boundary $\partial \mathcal{G}_{\varepsilon}^{-}$estimate

$$
\left\|(\lambda I-A)^{-1}\right\|_{E \rightarrow E} \leq M_{1} \cdot \varepsilon^{-1} \cdot(1+|\lambda|)^{-1}
$$

is true. Therefore for any analytic in the neighborhood of $\sigma(A)$ (scalar) function $\psi(z)$, such that esitmate

$$
(1+|z|)^{\alpha} \cdot|\psi(z)| \leq M_{2}
$$

takes place for some $0<\alpha<+\infty, 1 \leq M_{2}<+\infty$, the Cauchy-Riesz's formula defines bounded operator

$$
\psi(A)=\frac{1}{2 \pi \mathrm{i}} \int_{\partial \mathcal{G}_{\varepsilon}^{-}} \psi(z) \cdot(z I-A)^{-1} \mathrm{~d} z \quad(\mathrm{i}=\sqrt{-1}) .
$$

In particular, the negative fraction powers $A^{-\alpha}$ $(\alpha>0)$ of positive operator $A$ are defined (see [2]), $A^{-\alpha}=\left(A^{-1}\right)^{\alpha}$ for integer $\alpha$, and semigroup identity

$$
A^{-(\alpha+\beta)}=A^{-\alpha} \cdot A^{-\beta} \quad(0<\alpha, \beta<+\infty)
$$

is true. From these statements, evidently, it follows that positive fractional powers $A^{\alpha}(\alpha>0)$ can be defined by formula

$$
A^{\alpha}=\left(A^{-\alpha}\right)^{-1}
$$

Operators $A^{\alpha}(\alpha>0)$ already are unbounded, and their domains $D\left(A^{\alpha}\right)$ are dense in $E$. The following moment inequality

$$
\begin{gathered}
\left\|A^{\alpha} u\right\|_{E} \leq M(\alpha, \beta) \cdot\left\|A^{\beta} u\right\|_{E}^{\alpha / \beta} \cdot\|u\|_{E}^{1-\alpha / \beta} \\
{\left[0 \leq \alpha \leq \beta<+\infty, u \in D\left(A^{\beta}\right)\right]}
\end{gathered}
$$

takes place with some $1 \leq M(\alpha, \beta)<+\infty$, does not depend on $u \in D\left(A^{\beta}\right)$. Operator $A^{\alpha}$ for $\alpha \in(0,1)$ have the better spectral properties, than operator $A$. In particular, from identity

$$
\lambda I+A=(\sqrt{\lambda} I-\sqrt{A}) \cdot(\sqrt{\lambda} I+\sqrt{A}),
$$

inequality (1.19) (for $\alpha=\frac{1}{2}, \beta=1$ ) and estimate (1.14) it follows that operator $\sqrt{\lambda} I-\sqrt{A}$ has the bounded inverse for $\lambda \in \mathcal{G}_{\varepsilon}^{-}$, and estimate

$$
\left\|(\sqrt{\lambda} I-A)^{-1}\right\|_{E \rightarrow E} \leq M_{3} \cdot \varepsilon^{-1}(1+\sqrt{|\lambda|})^{-1}
$$

is true for some $1 \leq M_{3}<+\infty$, does not depend on $\epsilon$ and $\lambda$. In particular, operator $\lambda I+\sqrt{A}$ has bounded inverse for any complex number $\lambda$ such that $\operatorname{Re} \lambda \geq 0$, and estimate

$$
\left\|(\lambda I+\sqrt{A})^{-1}\right\|_{E \rightarrow E} \leq M \cdot(|\lambda|+1)^{-1}
$$

holds. Acting in the Banach space $E$ linear operator $B$ with dense domain $D(B)$ is called strongly positive (see [2]), if operator $\lambda I+B$ has bounded inverse for any complex number $\lambda$ with $\operatorname{Re} \lambda \geq 0$, and estimate

$$
\left\|(\lambda I+B)^{-1}\right\|_{E \rightarrow E} \leq M \cdot(|\lambda|+1)^{-1}
$$

is true for some $1 \leq M<+\infty$. Operator $B$ is strongly positive iff $-B$ is the generator of analytic semigroup $\exp \{-t B\}(t \geq 0)$ of linear bounded in $E$ operators with exponentially decreasing norm, when $t \rightarrow+\infty$, i.e. estimates

$$
\begin{aligned}
& \|\exp \{-t B\}\|_{E \rightarrow E}, \quad\|t B \cdot \exp \{-t B\}\|_{E \rightarrow E} \\
& \quad \leq M(B) \cdot \mathrm{e}^{-a(B) t} \quad(t>0)
\end{aligned}
$$

are true for some $1 \leq M(B)<+\infty, \quad 0<$ $a(B)<+\infty$. Thus, $\sqrt{A}$ is strongly positive in $E$ operator, i.e. the following estimates hold:

$$
\begin{aligned}
& \|\exp \{-t \sqrt{A}\}\|_{E \rightarrow E}, \quad \| t \sqrt{A} \cdot \exp \{-t \sqrt{A}\}_{E \rightarrow E} \\
& \leq M(\sqrt{A}) \cdot \mathrm{e}^{-a(\sqrt{A}) t} \quad(t>0) .
\end{aligned}
$$


The consideration of operator $\sqrt{A}$ permits to reduce differential equation (1.1) of the second order to equivalent system

$$
\begin{gathered}
v^{\prime}(t)+\sqrt{A} \cdot v(t)=z(t),-z^{\prime}(t)+\sqrt{A} \cdot z(t)=f(t) \\
(-\infty<t<+\infty)
\end{gathered}
$$

of differential equations of the first order. This fact prompts that for solution $v(t)$ in $C(E)$ of Eq. (1.1) formula

$$
v(t)=\frac{1}{2 \sqrt{A}} \int_{-\infty}^{+\infty} \exp \{-\sqrt{A}|t-s|\} \cdot f(s) \mathrm{d} s
$$

must be true. It is easy to see that formula (1.27) defines the unique solution in $C(E)$ of Eq. (1.1) if, for example.

$$
A f(t) \text { or } f^{\prime \prime}(t) \in C(E)
$$

It turns out that formula (1.27) defines the unique solution in $C(E)$ of Eq. (1.1) under essentially less restrictions on the smoothness of function $f(t)$.

\subsection{Well-Posedness in $C^{\alpha}(E)$}

We will consider differential equation (1.1) as the operator equation in the (abstract) Hölder space $C^{\alpha}(E)=C^{\alpha}[(-\infty,+\infty), E](0<\alpha<1)$ with norm

$$
\begin{aligned}
\|\psi\|_{C^{\prime}(E)}= & \sup _{-\infty<t<+\infty}\|\psi(t)\|_{E} \\
& +\sup _{-\infty<t<t+s<+\infty} \| \psi(t+s) \\
& -\psi(t) \|_{E} \cdot s^{-\alpha} .
\end{aligned}
$$

Analogously to the case of space $C(E)$ the notion of solution $v(t)$ of Eq. (1.1) in the space $C^{\alpha}(E)$ is defined. The well-posedness in $C^{\alpha}(E)$ of Eq. (1.1) means that coercive inequality

$$
\|v\|_{C^{\alpha}(E)}+\|A v\|_{C^{\alpha}(E)} \leq M(\alpha) \cdot\|f\|_{C^{\alpha}(E)}
$$

is true for its solution $v(t)$ in $C^{\alpha}(E)$ with some $1 \leq M(\alpha)<+\infty$, does not depend on $f(t) \in$ $C^{\alpha}(E)$. As in the case of space $C(E)$ it is established that from coercive inequality (1.30) the positivity of operator $A$ in Banach space $E$ follows. It turns out that this property of operator $A$ in $E$ is not only necessary, but also sufficient condition of well-posedness of Eq. (1.1) in $C^{\alpha}(E)$ for all $\alpha \in(0,1)$. In fact, from formula (1.27), evidently, it follows that

$$
\begin{aligned}
A v(t)= & \frac{\sqrt{A}}{2} \int_{-\infty}^{+\infty} \exp \{-\sqrt{A}|t-s|\} \\
& \times[f(s)-f(t)] \mathrm{d} s+f(t) .
\end{aligned}
$$

The application of estimates (1.25) leads us to estimate

$$
\begin{aligned}
\|A v(t)\|_{E} \leq & \frac{1}{2} \cdot M(\sqrt{A}) \\
& \times \int_{-\infty}^{+\infty} \mathrm{e}^{-a(\sqrt{A}) \cdot|t-s|} \cdot|t-s|^{\alpha-1} \mathrm{~d} s \\
& \times H^{\alpha}(f)+\|f\|_{C(E) .}
\end{aligned}
$$

Here and in what follows

$$
H^{\alpha}(f)=\sup _{-\infty<t<t+s<+\infty}\|f(t+s)-f(t)\|_{E} \cdot s^{-\alpha} .
$$

Formula (1.31) permits also to estimate Hölder coefficient $H^{\alpha}(A v)$ of function $A v(t)$. These estimates lead us to the following results:

THEOREM 1.1 Equation (1.1) is well-posed in functional Banach space $C^{\alpha}(E)(0<\alpha<1)$, iff $A$ is positive operator in Banach space E. For solution $v(t)$ in $C^{\alpha}(E)$ of Eq. (1.1) coercive inequality

$$
\|A v\|_{C^{\prime r}(E)} \leq M \cdot \alpha^{-1} \cdot(1-\alpha)^{-1} \cdot\|f\|_{C^{\prime}(E)}
$$

takes place with some $1 \leq M \leq+\infty$, does not depend on $f \in C^{\alpha}(E)$ and $\alpha \in(0,1)$. 


\section{DIFFERENCE EQUATION OF THE SECOND ORDER IN THE BANACH SPACE}

\subsection{Well-Posedness in $C^{h}(E)$}

Now we will consider the difference analog of differential equation (1.1), namely difference equation

$$
\begin{aligned}
& -\left(v_{i+1}-2 \cdot v_{i}+v_{i-1}\right) \cdot h^{-2}+A v_{i}=f_{i} \\
& \quad(i=\overline{-\infty,+\infty}) .
\end{aligned}
$$

Difference equation of the second order (2.1) is equivalent to system of difference equations of the first order

$$
\begin{aligned}
& \left(v_{i}-v_{i-1}\right) \cdot h^{-1}+\tilde{B} v_{i}=z_{i}, \\
& \quad-\left(z_{i+1}-z_{i}\right) \cdot h^{-1}+\tilde{B} z_{i}=(1+h \tilde{B}) f_{i} \\
& \quad(i=\overline{-\infty,+\infty})
\end{aligned}
$$

which is analogous to system of differential equation (1.26). Here operator $B=B\left(h^{2} A\right)=h \tilde{B}$ is defined by formula

$$
\left.B=h^{2} A / 2+\left[h^{2} A / 2\right)^{2}+h^{2} A\right]^{1 / 2},
$$

i.e. $B$ is the solution of operator quadratic equation

$$
B^{2} \cdot(1+B)^{-1}=h^{2} A \text {. }
$$

We will consider difference equation (2.1) as operator equation in the Banach space $C^{h}(E)$ of grid functions

$$
\psi^{h}=\left(\psi_{i} \in E ; i=\overline{-\infty,+\infty}\right)
$$

with norm

$$
\left\|\psi^{h}\right\|_{C^{h}(E)}=\sup _{i=-\infty,+\infty}\left\|\psi_{i}\right\|_{E}
$$

System (2.2) permits to show that for any $f^{h} \in C^{h}(E)$ there exists the unique solution $v^{h}$, $A v^{h}=\left(A v_{i}, i=\overline{-\infty,+\infty}\right) \in C^{h}(E)$ of Eq. (2.1), defining by formula

$$
\begin{gathered}
A v_{i}=B \cdot(2+B)^{-1} \cdot \sum_{k=-\infty}^{+\infty}(1+B)^{-|i-k|} f_{k} \\
(i=\overline{-\infty,+\infty}),
\end{gathered}
$$

which is analogous to formula (1.27). The basis of these statements will be given under supposition that $A$ is positive operator in $E$, and estimate (1.9) will be comfortable to write in the form

$$
\left\|(\lambda I+A)^{-1}\right\|_{E \rightarrow E} \leq M(A) \cdot[\lambda+a(A)]^{-1}
$$

for any $\lambda \geq 0$ and some $1 \leq M(A)<+\infty$, $0<a(A)<+\infty$. For the investigation of spectral properties of unbounded operator $B\left(h^{2} A\right)$ we will construct the bounded operator $[\lambda I+$ $\left.B\left(h^{2} A\right)\right]^{-1}$ for $\lambda \geq 0$. Since (scalar) function

$$
B(z)=z / 2+\left(z^{2} / 4+z\right)^{1 / 2}
$$

is analytic on whole complex plane, except points $0,-4$, and $B(z) \cdot z^{-1} \rightarrow 1$, when $|z| \rightarrow+\infty$, then, in virtue of estimate (2.8), the Cauchy-Riesz formula gives

$$
\begin{aligned}
& {\left[\lambda I+B\left(h^{2} A\right)\right]^{-1}} \\
& =\frac{1}{2 \pi \mathrm{i}} \int_{h^{2} \partial \mathcal{G}_{\varepsilon}^{-1}}[\lambda+B(z)]^{-1} \cdot\left(z I-h^{2} A\right)^{-1} \mathrm{~d} z \\
& \quad(\mathrm{i}=\sqrt{-1}) .
\end{aligned}
$$

Finally, since $z=0,-4$ are the bifurcation points of function $B(z)$, then the deformation of integration contour, in virtue of Cauchy's theorem, leads us to the formula

$$
\begin{aligned}
{[\lambda I+} & \left.B\left(h^{2} A\right)\right]^{-1} \\
= & \frac{1}{2 \pi} \int_{0}^{4}\left(\lambda^{2}-\lambda \rho+\rho\right)^{-1} \cdot \sqrt{\rho(4-\rho)} \\
& \times\left(\rho I+h^{2} A\right)^{-1} \mathrm{~d} \rho
\end{aligned}
$$


Since, evidently, function

$$
\begin{aligned}
& M_{1}(\lambda, \rho) \\
& \quad=(2 \pi)^{-1} \cdot\left(\lambda^{2}-\lambda \rho+\rho\right)^{-1} \cdot \sqrt{\rho(4-\rho)} \geq 0 \\
& \quad(0 \leq \rho \leq 4, \lambda \geq 0),
\end{aligned}
$$

then, in virtue of estimate (2.8), estimate

$$
\begin{aligned}
& \left\|\left[\lambda I+B\left(h^{2} A\right)\right]^{-1}\right\|_{E \rightarrow E} \leq M(A) \\
& \quad \times \int_{0}^{4} M_{1}(\lambda, \rho) \cdot\left[\rho+h^{2} a(A)\right]^{-1} \mathrm{~d} \rho
\end{aligned}
$$

is true. The application now of formulas (2.11) and (2.12) in the case, when operator $h^{2} A$ is replaced by number $h^{2} a(A)$, gives estimate

$$
\begin{aligned}
& \left\|\left[\lambda I+B\left(h^{2} A\right)\right]^{-1}\right\|_{E \rightarrow E} \\
& \quad \leq M(A) \cdot\left\{\lambda+B\left[h^{2} a(A)\right]\right\}^{-1} \\
& \quad \leq M(A) \cdot[\lambda+h \sqrt{a(A)}]^{-1} .
\end{aligned}
$$

Analogously to formula (2.11) for any $m=\overline{1,+\infty}$ formula

$$
\left[\lambda I+B\left(h^{2} A\right)\right]^{-m}=\int_{0}^{4} M_{m}(\lambda, \rho) \cdot\left(\rho I+h^{2} A\right)^{-1} \mathrm{~d} \rho
$$

is established. However, function $M_{m}(\lambda, \rho)$ for $m \geq 2$ changes the sign on segment $0 \leq \rho \leq 4$. Therefore the method, which was applied in the case $m=1$, does not work in the cases $m \geq 2$. We will suppose additionally that $-A$ is the generator of strongly continuous semigroup $\exp \{-t A\}(t \geq 0)$ with exponentially decreasing norm, i.e. estimate

$$
\|\exp \{-t A\}\|_{E \rightarrow E} \leq M(A) \cdot \mathrm{e}^{-t a(A)} \quad(t \geq 0)
$$

takes place for some $1 \leq M(A)<+\infty, 0<a$ $(A)<+\infty$. Then, it is well known (see [2]), there exists the bounded inverse $(\lambda I+A)^{-1}$ for any complex number $\lambda$ with $\operatorname{Re} \lambda>-a(A)$, and formula

$$
(\lambda I+A)^{-1}=\int_{0}^{+\infty} \mathrm{e}^{-\lambda t} \cdot \exp \{-t A\} \mathrm{d} t
$$

holds. Formula (2.17) means that resolvent of operator $-A$ is the Laplace transform of semigroup $\exp \{-t A\}$. From (2.16) and (2.17), in particular, it follows that $A$ is positive operator in $E$, i.e. estimate (2.8) is true.

Further from (2.15) it follows that

$$
\begin{gathered}
{\left[\lambda I+B\left(h^{2} A\right)\right]^{-m}=\int_{0}^{\infty} \mathcal{L}_{m}(\lambda, t) \cdot \exp \left\{-t h^{2} a\right\} \mathrm{d} t} \\
\mathcal{L}_{m}(\lambda, t)=\int_{0}^{4} M_{m}(\lambda, \rho) \cdot \mathrm{e}^{-\rho t} \mathrm{~d} \rho
\end{gathered}
$$

In the case when $h^{2} A$ is the positive numbers, formula (2.18) means that function $\left[\lambda I+B\left(h^{2} A\right)\right]^{-m}$ is (for fixed $\lambda \geq 0$ ) the Laplace transform of function $\mathcal{L}_{m}(\lambda, t)$. Then from properties of Laplace transform it follows that $\mathcal{L}_{m}(\lambda, t)$ is the convolution of $m$ copies of function $\mathcal{L}_{1}(\lambda, t)$, and this convolution is defined by recurrent correlation

$$
\begin{gathered}
\mathcal{L}_{m+1}(\lambda, t)=\int_{0}^{t} \mathcal{L}_{m}(\lambda, s) \cdot \mathcal{L}_{1}(\lambda, t-s) \mathrm{d} s \\
(m=\overline{1,+\infty}) .
\end{gathered}
$$

Since $M_{1}(\lambda, \rho) \geq 0$, then from (2.19) it follows that $\mathcal{L}_{1}(\lambda, \rho) \geq 0$. Therefore, in virtue of (2.20),

$$
\mathcal{L}_{m}(\lambda, t) \geq 0 \quad(m=\overline{1,+\infty}) .
$$

Inequality (2.21) permits to apply by estimate of norm of operator $\left[\lambda I+B\left(h^{2} A\right)\right]^{-m}$, defining by formula (2.18), the same approach, as in the case $m=1$ for formula (2.11). Namely estimate $(\lambda \geq 0)$

$$
\begin{aligned}
\| & {\left[\lambda I+B\left(h^{2} A\right)\right]^{-m} \|_{E \rightarrow E} } \\
& \leq M(A) \cdot\left\{\lambda+B\left[h^{2} a(A)\right]\right\}^{-m} \\
& \leq M(A) \cdot[\lambda+h \sqrt{a(A)}]^{-m}
\end{aligned}
$$


is true. This estimate permits to establish that difference equation (2.1) is well-posed in the space $C^{h}(E)$ (for fixed $h$ ), i.e. for any $f^{h} \in C^{h}(E)$ there exists the unique solution in $C^{h}(E)$ of Eq. (2.1), defining by formula (2.7), and following inequalities

$$
\begin{aligned}
\left\|v^{h}\right\|_{C^{h}(E)} & \leq M_{s}(h) \cdot\left\|f^{h}\right\|_{C^{h}(E)}, \\
\left\|A v^{h}\right\|_{C^{h}(E)} & \leq M_{C}(h) \cdot\left\|f^{h}\right\|_{C^{h}(E)}
\end{aligned}
$$

are true with some $1 \leq M_{s}(h), M_{C}(h)<+\infty$, do not depend on $f^{h} \in C^{h}(E)$. Inequality (2.23) is, evidently, corollary of inequality (2.24), since $A^{-1}$ is bounded operator in $E$.

However under investigation of convergence of difference method it is necessary to establish the well-posedness of Eq. (2.1) in Banach space $C^{h}(E)$ not for some fixed $h \in(0,1)$ but in the aggregate of such spaces for all $h \in(0,1]$. To this aim we must establish inequalities

$$
\begin{aligned}
\left\|v^{h}\right\|_{C^{h}(E)} & \leq M_{s} \cdot\left\|f^{h}\right\|_{C^{h}(E)} \\
\left\|A v^{h}\right\|_{C^{h}(E)} & \leq M_{C}\left\|f^{h}\right\|_{C^{h}(E)}
\end{aligned}
$$

with some $1 \leq M_{s}, M_{c}<+\infty$, do not depend on $f^{h} \in C^{h}(E)$ and $0<h \leq 1$. Inequality (2.26) is, generally speaking, not true for any Banach space $E$ and generator $-A$ of strongly continuous semigroup $\exp \{-t A\}$ with exponentially decreasing norm, and this statement (see Section 1.1) follows from ill-posedness in $C(E)$ of Eq. (1.1).

It turns out that the more weaker inequality (2.25) is true. Namely formula (2.7) and estimate (2.22) permit to show that we can put

$$
\begin{aligned}
M_{s}= & M(A) \cdot[a(A)]^{-1 / 2} \cdot[1+M(A)]^{1 / 2} \\
& \times\left\{1+2[a(A)]^{-1 / 2}\right\} .
\end{aligned}
$$

The property (2.25) is called the stability of difference equation (2.11) in the Banach space $C^{h}(E)$.

\subsection{Well-Posedness in $C^{h, \alpha}(E)$}

Now we want to consider difference equation (2.1) as operator equation in the Banach space $C^{h, \alpha}(E) \quad(0<\alpha<1)$ of grid functions $\psi^{h}=$ $\left(\psi_{i} \in E ; i=\overline{-\infty,+\infty}\right)$ with norm

$$
\begin{aligned}
\left\|\psi^{h}\right\|_{C^{h, \alpha}(E)} & =\sup _{i=-\infty,+\infty}\left\|\psi_{i}\right\|_{E} \\
& +\sup _{-\infty<i<i+k<+\infty}\left\|\psi_{i+k}-\psi_{i}\right\|_{E} \cdot(k h)^{-\alpha} .
\end{aligned}
$$

The well-posedness of difference equation (2.1) in the aggregate of such space for all $h \in(0.1]$ means that for solutions $v^{h}$ of Eq. (2.1) in $C^{h, \alpha}(E)$ stability inequality

$$
\left\|v^{h}\right\|_{C^{h, \alpha}(E)} \leq M_{s}(\alpha) \cdot\|f\|_{C^{h, \alpha}(E)}
$$

and coercive inequality

$$
\left\|A v^{h}\right\|_{C^{h, \alpha}(E)} \leq M_{C}(\alpha) \cdot\left\|f^{h}\right\|_{C^{h, \alpha}(E)}
$$

are true for some $1 \leq M_{s}(\alpha), M_{C}(\alpha)<+\infty$, do to depend on $f^{h} \in C^{h, \alpha}(E)$ and $h \in(0,1]$.

The stability in $C^{h, 1}(E)$, evidently, from stability in $C^{h}(E)$ follows. Then the application of interpolation theorem permits to estabish the stability in the space $C^{h, \alpha}(E)$ for all $\alpha \in(0,1]$.

Further we will suppose that $A$ is strongly positive in $E$ operator, i.e. $-A$ is the generator of analytic semi-group with exponentially decreasing norm:

$$
\begin{aligned}
& \|\exp \{-t A\}\|_{E \rightarrow E}, \quad\|t A \cdot \exp \{-t A\}\|_{E \rightarrow E} \\
& \quad \leq M(A) \cdot \mathrm{e}^{-t a(A)} \\
& \quad(t>0), 1 \leq M(A)<+\infty, 0<a(A)<+\infty .
\end{aligned}
$$

The proof of well-posedness of difference equation (2.1) in $C^{h, \alpha}(E)$, i.e. the proof of coercive 
inequality (2.29), is based on estimates of norms of operators.

$$
\begin{aligned}
& B \cdot(2+B)^{-1} \cdot(1+B)^{-m}, \\
& B^{2} \cdot(2+B)^{-1} \cdot(1+B)^{-m} \quad(m=\overline{1,+\infty})
\end{aligned}
$$

Analogously to formula (2.18) we can establish formula

$$
\begin{aligned}
{\left[2+B\left(h^{2} a\right)\right]^{-1} \cdot\left[1+B\left(h^{2} A\right)\right]^{-m} } \\
\quad=\int_{0}^{-\infty} \mathcal{L}_{1}(2, t) * \mathcal{L}_{m}(1, t) \cdot \exp \left\{-t h^{2} A\right\} \mathrm{d} t .
\end{aligned}
$$

Here $\mathcal{L}_{1}(2, t) * \mathcal{L}_{m}(1, t)$ is the convolution of functions $\mathcal{L}_{1}(2, t)$ and $\mathcal{L}_{m}(1, t)$. Formula (2.32), evidently, leads us to estimate

$$
\begin{aligned}
& \left\|(2+B)^{-1} \cdot(1+B)^{-m}\right\|_{E \rightarrow E} \\
& \quad \leq M(A) \cdot\left\{1+h[a(A)]^{1 / 2}\right\}^{-(m+1)},
\end{aligned}
$$

which is analogous to estimate (2.22). Further, in virtue of formulas (2.4) and (2.32), we have for $m \geq 2$ formula

$$
\begin{aligned}
B^{2}(2 & +B)^{-1} \cdot(1+B)^{-m} \\
= & \int_{0}^{+\infty} \mathcal{L}_{1}(2, t) * \mathcal{L}_{m-1}(1, t) \\
& \times h^{2} A \cdot \exp \left\{-t h^{2} A\right\} \mathrm{d} t .
\end{aligned}
$$

Now we apply estimate (2.30) and obtain

$$
\begin{aligned}
&\left\|B^{2} \cdot(2+B)^{-1} \cdot(1+B)^{-m}\right\|_{E \rightarrow E} \\
& \leq M(A) \cdot \int_{0}^{+\infty} \mathcal{L}_{1}(2, t) * \mathcal{L}_{m-1}(1, t) \\
& \quad \times t^{-1} \cdot \mathrm{e}^{-t h^{2} a(A)} \mathrm{d} t .
\end{aligned}
$$

Further from evident formula

$$
t^{-1}=\int_{0}^{+\infty} \mathrm{e}^{-t s} \mathrm{~d} s \quad(t>0)
$$

it follows that

$$
\begin{aligned}
&\left\|B^{2} \cdot(2+B)^{-1} \cdot(1+B)^{-m}\right\|_{E \rightarrow E} \\
& \leq M(A) \cdot \int_{0}^{+\infty}\left\langle\int_{0}^{+\infty} \mathcal{L}_{1}(2, t) * \mathcal{L}_{m-1}(1, t)\right. \\
&\left.\quad \times \mathrm{e}^{-t\left[s+h^{2} a(A)\right]} \mathrm{d} t\right\rangle \mathrm{d} s .
\end{aligned}
$$

Finally, the application of formula (2.32) for the case, when operator $h^{2} A$ is replaced by number $s+h^{2} a(A)$, gives estimate

$$
\begin{aligned}
&\left\|B^{2} \cdot(2+B)^{-1} \cdot(1+B)^{-m}\right\|_{E \rightarrow E} \\
& \leq M(A) \cdot \int_{0}^{+\infty}\left\{2+B\left[s+h^{2} \cdot a(A)\right]\right\}^{-1} \\
& \quad \times\left\{1+B\left[s+h^{2} \cdot a(A)\right]\right\}^{-(m-1)} \mathrm{d} s .
\end{aligned}
$$

Therefore the following estimate is true:

$$
\begin{gathered}
\left\|B^{2}\left(h^{2} A\right) \cdot\left[2+B\left(h^{2} A\right)\right]^{-1} \cdot\left[1+B\left(h^{2} A\right)\right]^{-m}\right\|_{E \rightarrow E} \\
\quad \leq 2 M(A) \cdot\left(m^{2}-1\right)^{-1} \leq M_{2} \cdot m^{-2} .
\end{gathered}
$$

For operator $B \cdot(2+B)^{-1} \cdot(1+B)^{-m}(m \geq 2)$ we apply estimate (2.37) and (2.39), moment inequality

$$
\|B \psi\|_{E} \leq M_{B} \cdot\left\|B^{2} \psi\right\|_{E}^{1 / 2} \cdot\|\psi\|_{E}^{1 / 2}\left[\psi \in D\left(B^{2}\right)\right]
$$

and obtain

$$
\begin{aligned}
& \left\|B\left(h^{2} A\right) \cdot\left[2+B\left(h^{2} A\right)\right]^{-1} \cdot\left[1+B\left(h^{2} a\right)\right]^{-m}\right\|_{E \rightarrow E} \\
& \quad \leq M_{1} \cdot m^{-1} .
\end{aligned}
$$

It is easy to see that estimates (2.38) and (2.41) are true also in the case $m=1$. These estimates are analogous to estimates of analytic semigroup. They permit to establish the following result.

THEOREM 2.1 Let A be strongly positive operator in the Banach space E. Then difference equation (2.1) is well-posed in the aggregate of Banach 
space $C^{h, \alpha}(E)(0<\alpha<1)$ and coercive inequality

$$
\left\|A v^{h}\right\|_{C^{h, \alpha}(E)} \leq M \cdot \alpha^{-1} \cdot(1-\alpha)^{-1} \cdot\left\|f^{h}\right\|_{C^{h, \alpha}(E)}
$$

holds for its solutions $v^{h}$ in $C^{h, \alpha}(E)$ with some $1 \leq M<+\infty$, does not depend on $f^{h} \in C^{h, \alpha}(E)$, $\alpha \in(0,1)$ and $h \in(0,1]$.

From definition (2.27) and from inequality (2.42), evidently, it follows that

$$
\left\|A v^{h}\right\|_{C^{h}(E)} \leq M \cdot \alpha^{-1} \cdot h^{-\alpha} \cdot\left\|f^{h}\right\|_{C^{h}(E)}
$$

for any $\alpha \in(0,1 / 2], h \in\left(0, \mathrm{e}^{-2}\right]$ and some $1 \leq$ $M<+\infty$, does not depend on $f^{h}, h$ and $\alpha$. We will put here

$$
\alpha=(\ln 1 / h)^{-1}
$$

and obtain

$$
\left\|A v^{h}\right\|_{C^{h(E)}} \leq M \cdot \mathrm{e} \cdot \ln 1 / h \cdot\left\|f^{h}\right\|_{C^{h}(E)} .
$$

It means that we can put

$$
M_{c}(h)=M \cdot \mathrm{e} \cdot \ln 1 / h
$$

in inequality (2.24).

\section{References}

[1] Allaberen Ashyralyev and Pavel E. Sobolevskii, Wellposedness of Parabolic Difference Equations. Birkhäuser, 1994, p. 346.

[2] M. A. Krasnoselskii et al., Integral Operators in Spaces of Summable Functions, Noordhoff International Publishing, 1976, p. 520.

[3] P. Grisvard, Equations differentielles abstraites, Ann. Scient. Ecole Norm. Super. 2(3) (1970) 68-125. 


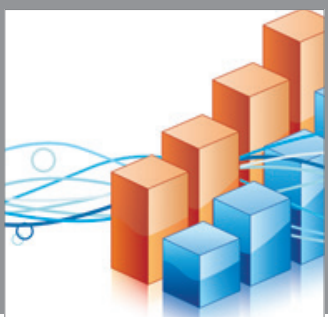

Advances in

Operations Research

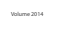

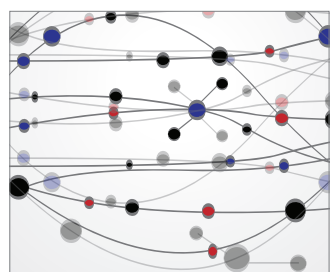

\section{The Scientific} World Journal
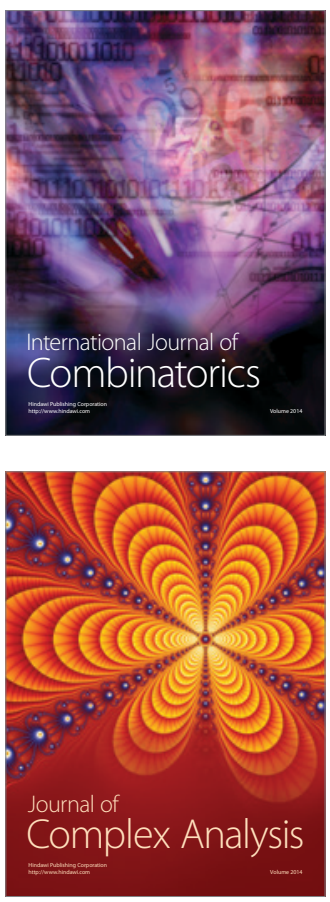

International Journal of

Mathematics and

Mathematical

Sciences
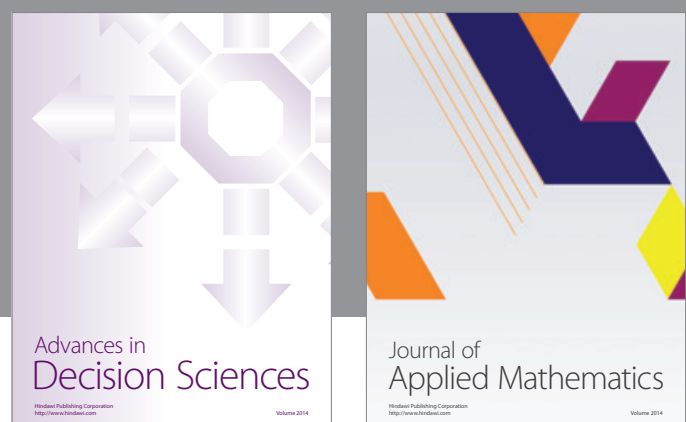

Journal of

Applied Mathematics
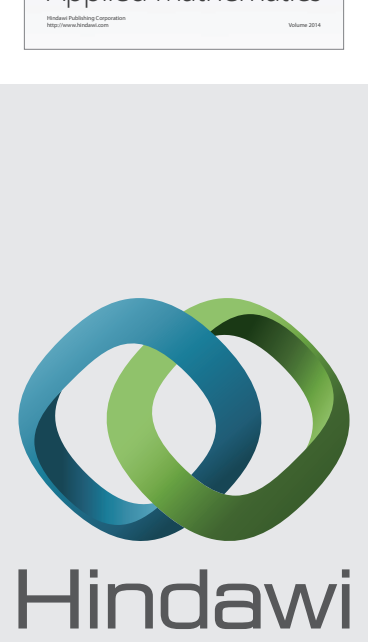

Submit your manuscripts at http://www.hindawi.com
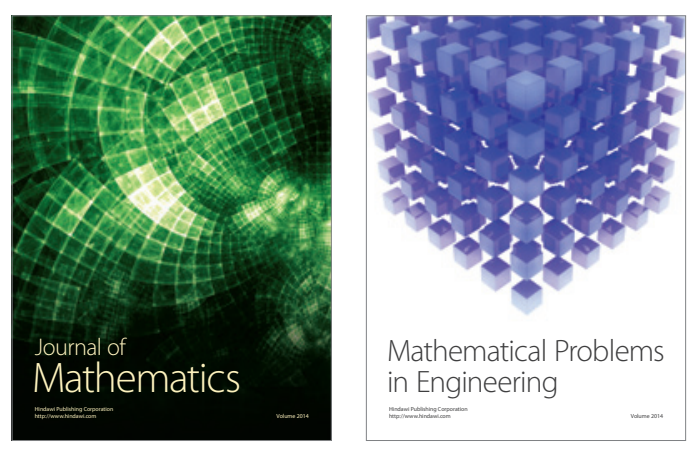

Mathematical Problems in Engineering
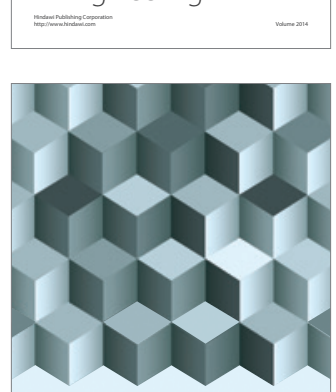

Journal of

Function Spaces
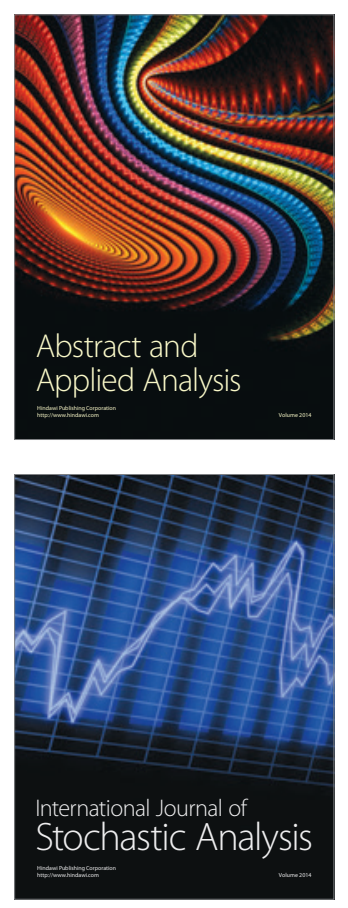

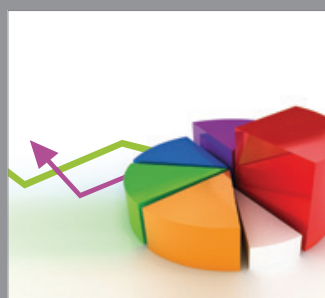

ournal of

Probability and Statistics

Promensencen
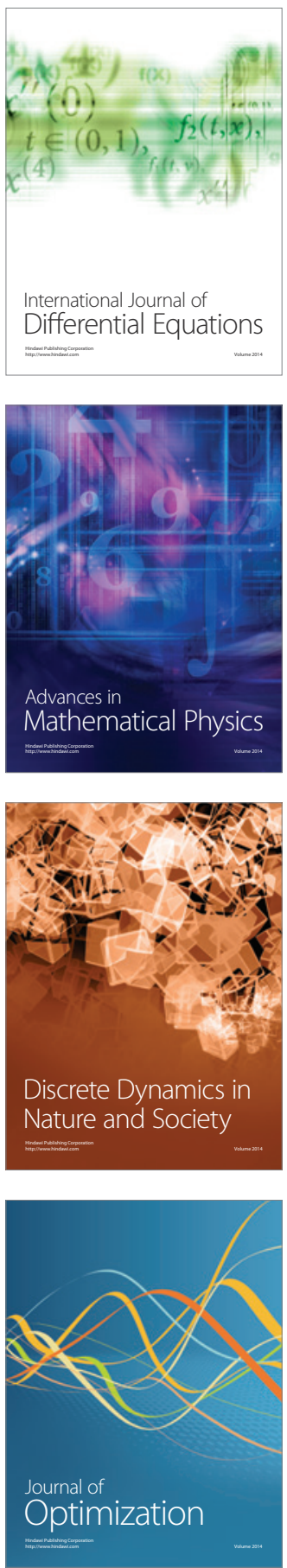